\title{
Optimising OSPF Database by Intra-Area Summarization: A New Approach
}

\author{
Deepak Malik \\ M.tech Scholar CSE, AITM \\ 70 KM, Delhi-Mathura Road \\ Aurangabad, Palwal, HR
}

\author{
Shikha Pandit \\ Asstt. Prof. M.tech CSE, AITM \\ 70 KM, Delhi-Mathura Road \\ Aurangabad, Palwal, HR
}

\author{
Pritam Kumar \\ M.tech Scholar CSE, ACTM \\ $70 \mathrm{KM}$, Delhi-Mathura Road \\ Aurangabad, Palwal, HR
}

\begin{abstract}
With the growing demand of communication, the need for networking is increasing day by day. Networking being the backbone of computer industry faces the greatest challenge. Factors like cost, time and memory size utilization are always the bottle neck for this industry. Various methods are available for dealing this problem. Protocols like EIGRP, OSPF, RIP, BGP, etc. are used in industry for establishing a network nowadays. These protocols have different working methods but the primary goal of all these routing protocols is the same. The aim is to calculate a path with a least cost so as to send traffic (both data and voice) via that path. This paper suggests and purposes a new technique for OSPF protocol working, for saving the database size and reducing routing table size resulting in better convergence time.
\end{abstract}

\section{General Terms}

Routing Protocols, Internetworking, Convergence time, CPU Utilization, Memory size.

\section{Keywords}

OSPF, OSPF area, LSA, LSDB, Inter-area, Intra-area, IOS, Router configuration

\section{INTRODUCTION}

In the world of networking there are two types of routing protocols used. They are either interior or exterior gateway protocols i.e. IGP and EGP respectively. RIP, OSPF, EIGRP are examples of IGP while Border Gateway Protocol (BGP) is an example of EGP. These protocols are responsible for the transportation of data of applications across a network such as IPX, IP, IPV6, etc. Each protocol is based on various algorithms which in turn depend on metrics (cost) to find the best path for sending data like cost, bandwidth, MTU, distance, etc. This paper discusses a new approach for OSPF working.

Open Shortest Path First (OSPF) is an open standard protocol. It is a Link-State routing protocol i.e. each node (router) has complete information about its neighboring networks and doesn't really on its neighboring advertisements as like Distance-Vector Principle. Each node has the complete topology information within that network. Each node uses this information to form a local database to manage and learn the topology for that region. The information is exchange with all neighboring nodes (routers) globally so that all the nodes within that particular region have a similar set of database called the topology information. Any change in the network is triggered updated i.e. whenever there is a change in topology occurs, instantly that information is passed on to all the neighboring routers for updating their topology database. The triggered updating helps in forming a single synchronized database of that region in each router present in that domain [1] [4].

\section{CURRENT WORKING OF OSPF}

OSPF being a Link-State routing protocol and uses LinkState-Advertisements (LSA) for passing information from one router to another. Information is passed only when neighbor ship is formed else not. The neighbor ship is formed only when these parameters like same subnet, same area, same Kvalues, etc. are matched. When these parameters are meeting up to a level, then only routers are said to be OSPF neighbors. Once the neighbor ship is formed second step, topology database exchange, requires each OSPF router to cooperate by sending messages so that all routers learn topology information that is the equivalent of the kinds of information a human would draw and write in a diagram of the internetwork. Each router stores this topology information in its topology database called its Link-State Database (LSDB). The information communicated by OSPF routers and held in their LSDB includes all the information required by a router to work with [2] [3].

Third important step, route computation, means that each router independent analysis the topology to choose the best routes from their perspective. Shortest Path First (SPF) Algorithm is used for each to determine the shortest (best) route for each reachable subnet, add the correct next outgoing destination routes in their routing table.

OSPF uses hierarchical design structure for working using OSPF areas. Each router present in one or more areas, depending upon the working requirement of that router. The hierarchical structure can be represented as shown in figure1.

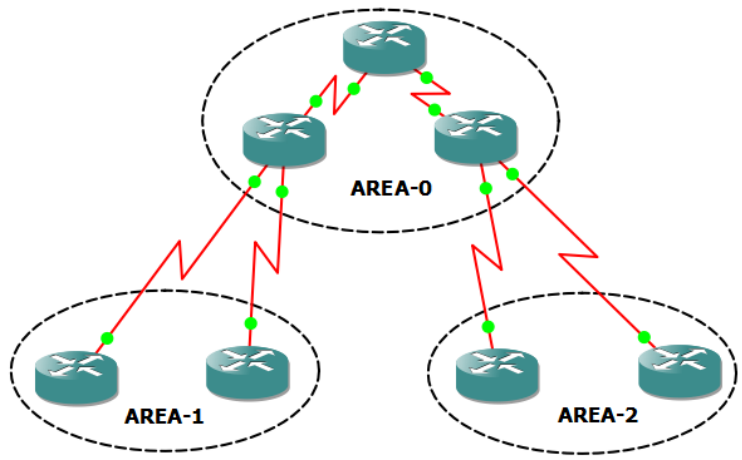

Figure 1: Hierarchical Structure of OSPF

There are various types of router classified on the working style in OSPF areas. These can be represented as:

1. Area Boundary Router (ABR): routers with their interfaces present in more than one area, including 
the backbone area. ABRs hold topology data for each area, and calculate routes for each area, and advertise about those routes between areas.

2. Autonomous System Boundary Router (ASBR): those routers whose interfaces are present in to different domains e.g. one in OSPF and other interface in EIGRP, etc.

3. Back Bone Router (BB): backbone routers with all interfaces present in area- 0 or Back Bone area

4. Internal Router: a router that has interfaces connected to only one area making the router completely internal to that one area.

5. Designated Router: on multiple access data links like LAN an OSPF router elected by the routers on that data link to perform special functions. These functions include the generation of LSA respectively the subnet, and playing key role in database exchange process.

6. Backup Designated Router (BDR): a router on a multi-access data link that monitors the DR and becomes prepared to take over for the DR, should the DR fail.

These OSPF routers can be represented as shown in figure 2

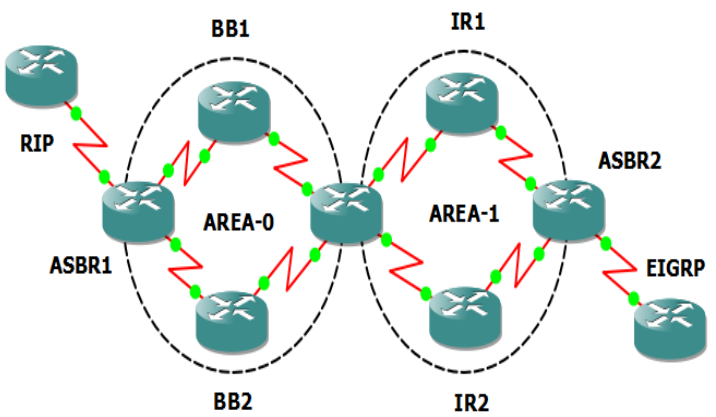

Figure 2: Types of OSPF Routers

There are some common terms used by OSPF as follows:

a. Link-State-Database (LSDB): the data structure hold by on OSPF router for the purpose of storing topology data.

b. Shortest-Path-First (SPF): the name of the algorithm OSPF uses to analyze the LSDB. The analysis determines the best (lowest cost) route for each prefix/ length.

c. Link-State-Update (LSU): the name of the OSPF packet that holds the detailed topology information, especially LSAs.

d. Link-State-Advertisement (LSA): the name of a class of OSPF data structure that hold topology information. LSAs are held in memory in the LSDB and communicated over the network in LSU messages.

e. AREA: a contiguous grouping of routers and router interfaces. Routers in an area strive to learn all topology information about the area, but they do not learn topology information about areas to which they do not connect.

Inside an area, router exchange detailed topology information. The detailed topology information doesn't flow between areas. Instead, the ABRs advertise briefer information between areas, including information about subnets/masks, but the information advertised into one area doesn't include details about the topology of the other area.

One area called the backbone area must be connected to all areas. Packets that need to pass between two non backbone areas must pass through (at least) one backbone router. The ABRs must keep a copy of the LSDB for each area to which they attach. However, the ABRs do not forward all the topology details between areas; instead they simply advertise the subnets (prefix/length) between the areas.

Table 1: Types of LSAs used in OSPF

\begin{tabular}{|l|l|l|}
\hline $\begin{array}{l}\text { LSA } \\
\text { Type }\end{array}$ & $\begin{array}{l}\text { Common } \\
\text { Name }\end{array}$ & Description \\
\hline 1 & Router & $\begin{array}{l}\text { Created by each router to represent } \\
\text { itself for each area to which it } \\
\text { connects, one Type-1 LSA per } \\
\text { router per area. }\end{array}$ \\
\hline 2 & Network & $\begin{array}{l}\text { One per transit area, created by DR } \\
\text { on the subnet. }\end{array}$ \\
\hline 3 & $\begin{array}{l}\text { Net } \\
\text { Summary }\end{array}$ & $\begin{array}{l}\text { Created by ABRs to represent } \\
\text { subnets listed in one area's type 1 } \\
\text { and 2 LSAs when being advertised } \\
\text { into another area. }\end{array}$ \\
\hline 4 & $\begin{array}{l}\text { ASBR } \\
\text { Summary }\end{array}$ & $\begin{array}{l}\text { Like a type 3 LSA, except it } \\
\text { advertises a host route used to } \\
\text { reach an ASBR. }\end{array}$ \\
\hline 5 & AS External & $\begin{array}{l}\text { Created by ASBRs for external } \\
\text { routes injected into OSPF. }\end{array}$ \\
\hline 6 & $\begin{array}{l}\text { Group } \\
\text { Membership }\end{array}$ & $\begin{array}{l}\text { Defined for MOSPF i.e. Multi } \\
\text { OSPF. }\end{array}$ \\
\hline 7 & NSSA & $\begin{array}{l}\text { Created by ASBRs inside an } \\
\text { NSSA area, instead of a type 5 } \\
\text { LSA. }\end{array}$ \\
\hline
\end{tabular}

\section{CURRENT SOLUTION FOR REDUCING MEMORY UTILIZATION IN OSPF}

\subsection{Limiting number of LSAs}

Due to link-state logic each OSPF router creates LSA for each network in the area it belongs and stores them in its local database. Complete network information is stored in each router database over the network, results a redundancy of information of LSAs. Large amount of memory size is thus required to store this information. Large information again takes more CPU utilization for processing and also increases the size of routing table of router. Thus, OSPF comes with the solution to divide the network size in small parts called areas. These areas further classified as Stub, Totally Stub, Not-SoStubby-Area (NSSA) and Totally NSSA. These can be implemented as over ABRs.

$>$ For all types of stubby areas, the ABR always filters Type 5 (external) LSAs.

$>$ For totally stubby and totally NSSA areas, the ABR also filters Type 3 LSAs. 
For stubby and NSSA areas-those without word "totally" in the name, the ABRs don't filter Type 3 LSAs as normal.

\subsection{Route Summarization}

OSPF allows summarization at ABRs and ASBRs but not on other OSPF routers. The main reason is again that the LSDB must be the same for all routers in a single area. So summarization can be done only on these two routers. These can only be implemented by doing manual summarization on these routers as OSPF don't have mechanism for AutoSummarization.

Both these above methods only helps in reducing the database size for some extend. But the routing table size is still large for router to compute. The memory and CPU utilization is also large due to no mechanism for LSA Type-1 and Type-2 summarization. Thus, this paper presents a solution for further reduce this problem up to much extend.

\section{NEW PROPOSAL FOR OSPF INTRA- AREA SUMMARIZATION}

The summary route advertised by an OSPF router within an area (i.e. intra-area) should be flooded to all OSPF routers including $\mathrm{ABR}$. But other OSPF routers only receives the summary route, while $A B R$ receives all the information i.e. summary route plus the routes in LSA-1 (all link-counts) with LSA-1 router-id corresponding to each router within that area, so that inter-area query scope could be summarize i.e. queryscope for inter-area for that summary route will be reduced. Thus, save time and traffic (bandwidth) for unnecessary travelling of packet to that router and then discard by particular OSPF router if route is not present within the advertised summary route of that router as shown

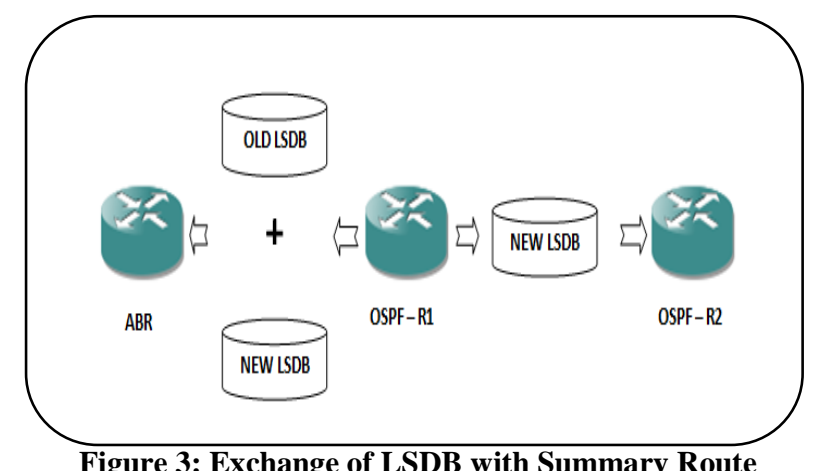

By combining concept of EIGRP route summarization over OSPF protocol one can reduce the problem of OSPF intraarea route summarization up to much extends.

Route summarization for type-1 and type-2 LSAs with in an area helps in reducing the database size as well as routing table per router per area. Thus, each router summarizes the routes by itself i.e. each router summarizes the routes present in its own type-1 LSA so that it can advertise this summary route to others OSPF neighbor routers. Summarization can be done by OSPF automatically as well as supports manual summarization as OSPF on ASBRs and ABRs as well.

This can be represented with the help of flowchart as:

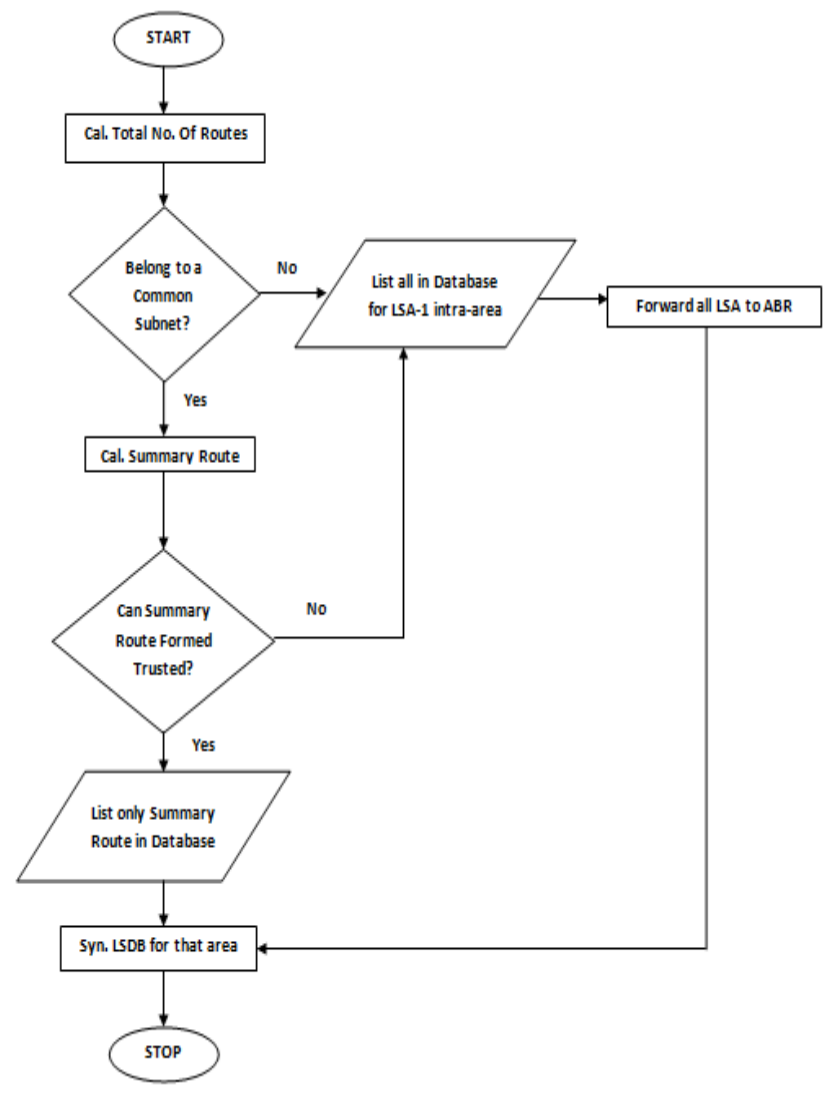

The LSA exchange only carry the summary route to other OSPF neighbor routers, rather than complete network information type-1 LSA and router with in an area. This summary route should be present in LSDB of all routers within that area. Each router thus calculate the SPF process on that summary route and stores the lowest cost (metric) into its routing table along with advertise router-id.

The routing table for ABR must contain all the routes within the network so as to have a better connectivity between different areas. This will surely not reduce the ABR overhead but will help in saving unwanted traffic flowing through the network if route is not present in summary route generated by a particular OSPF router. If a request is generated for a particular route, which falls within range of summary route, router forward that request to that particular advertised router for that summary route. If network is present with that advertised router, it should respond it else, it discards that request by an error message indicating no such route found. But this type of route summarization can only be done if we trust that summary route for a given network else it may lead to sub-optimal routing in the network.

Thus, rather than advertising different LSA routes (networks) OSPF can only advertise a single LSA or a single route over the routers in that area. Single LSA or route not only reduces routing table size for each router but also reduces the OSPF database i.e. Topology Table by a significant value. CPU consumption will also be less than before; thus, saving power as well as CPU utilization of that OSPF process.

\section{EXPERIMENTAL ANALYSIS}

In this analysis 2 different networks have same topologies. One is configured with EIGRP, the second one with OSPF. The common network topology is shown 


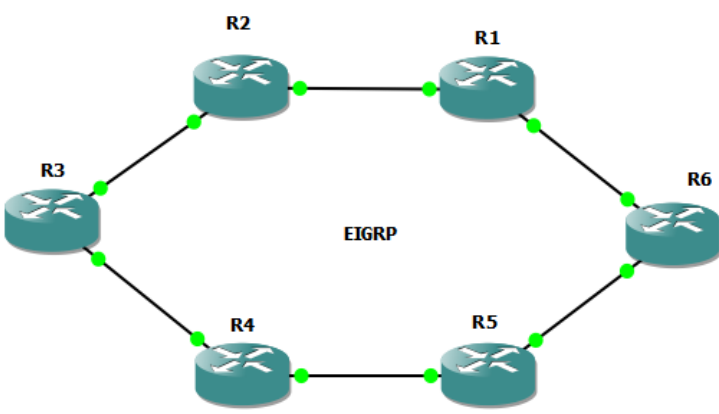

Figure 4: EIGRP network topology

There are six routers with six loopbacks networks each. Therefore in a total we have thirty-six networks along with six links in between each router. OSPF also has the same network topology as shown

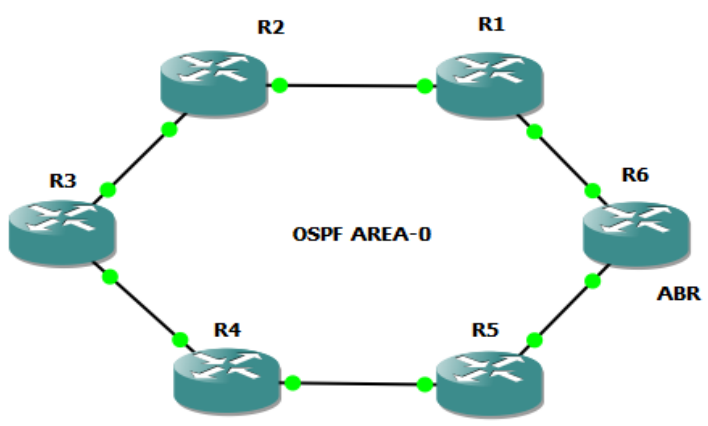

Figure 5: OSPF network topology

From the experiment it is observed that before summarization, routing table generated for both protocols whether EIGRP or OSPF, have same size on router memory. The routing size formed is independent of protocol used but it depends upon the number of links or networks used by the protocol used.

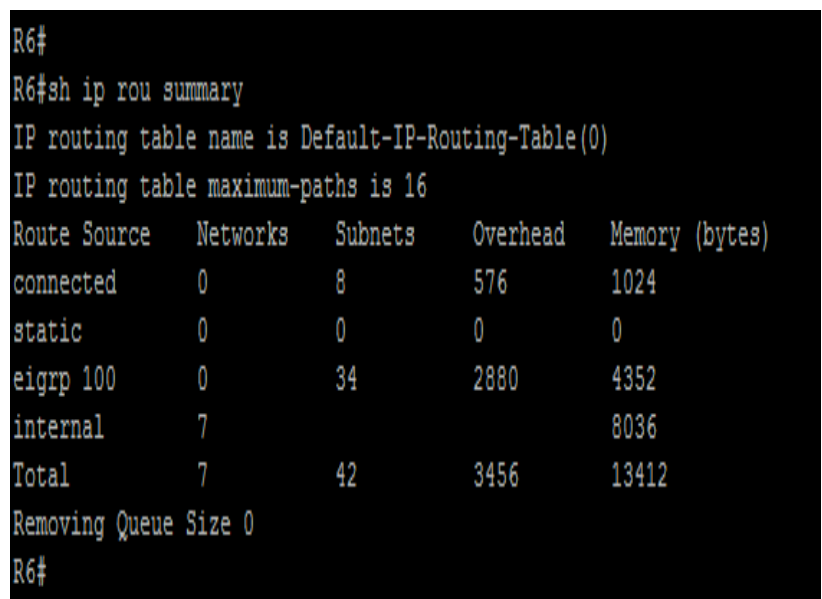

Figure 6: SNAPSHOTS of Routing Table for EIGRP before route summary

The table shows the 13412 bytes of memory used by EIGRP for storing 42 different networks subnets. Also OSPF uses the same memory for storing same topology as shown

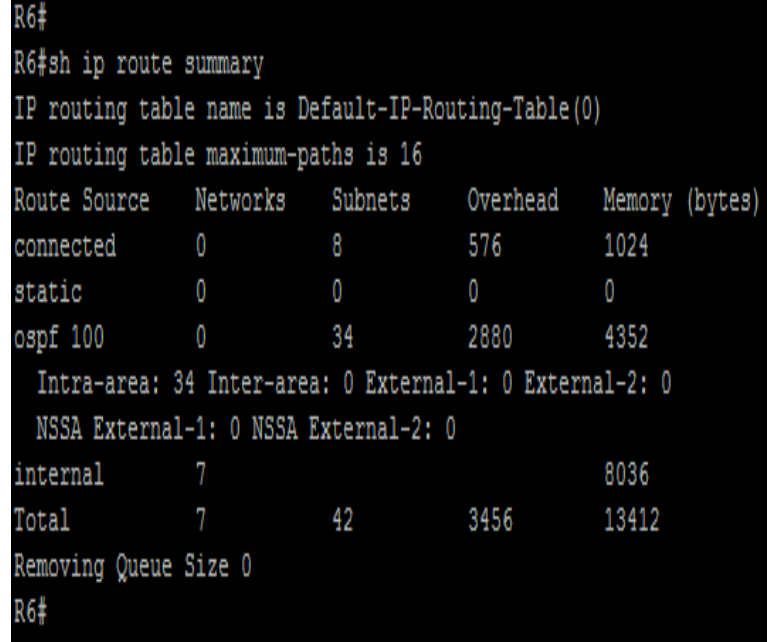

Figure 7: SNAPSHOTS of Routing Table for OSPF before route summary

But when EIGRP route summarization is done on each router, the routing table size is decreased to 10,340 bytes as shown

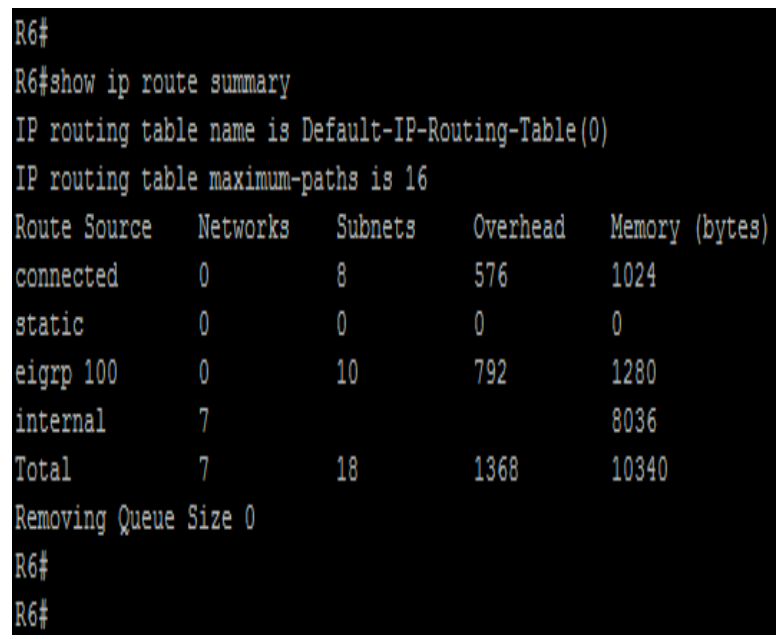

Figure 8: SNAPSHOTS of Reduced Routing Table after summarization

The routing table formed for the same topology has reduced size by almost $30 \%$ as compared to before summarization. Thus, result obtain can be shown in figure 9 .

OSPF uses router CPU for computing SPF process in large amount. By route summarization the CPU Utilization will also reduced to much extend as shown in figure 10 .

The Intra-area route summarization as well as Intra-area LSA summarization will also help in saving Hardware Cost due to lower consumption of memory. 


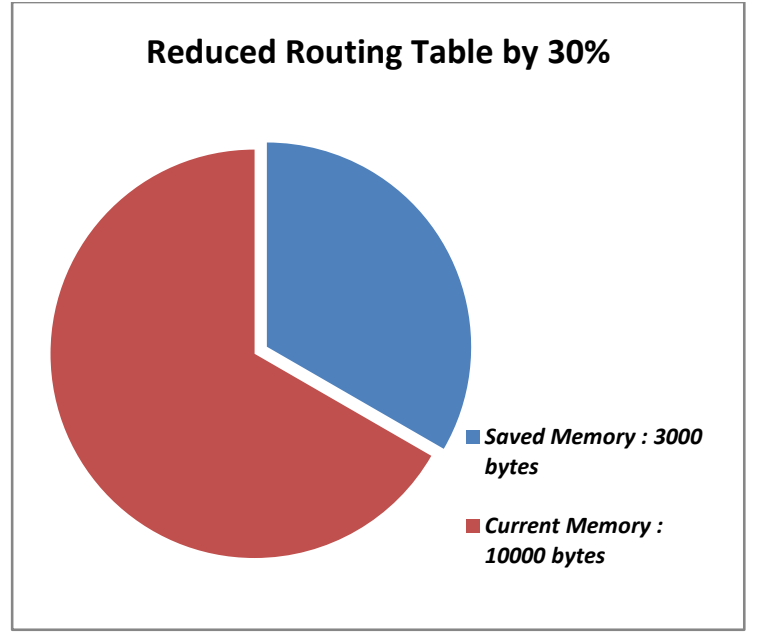

Figure 9: Memory Status after Route Summarization

The Intra-area summarization in OSPF also has a significant impact on the convergence time i.e. making is more easily converge with in an area. Thus, summarization also helps in improving the convergence time which is a significant parameter for a routing protocol.

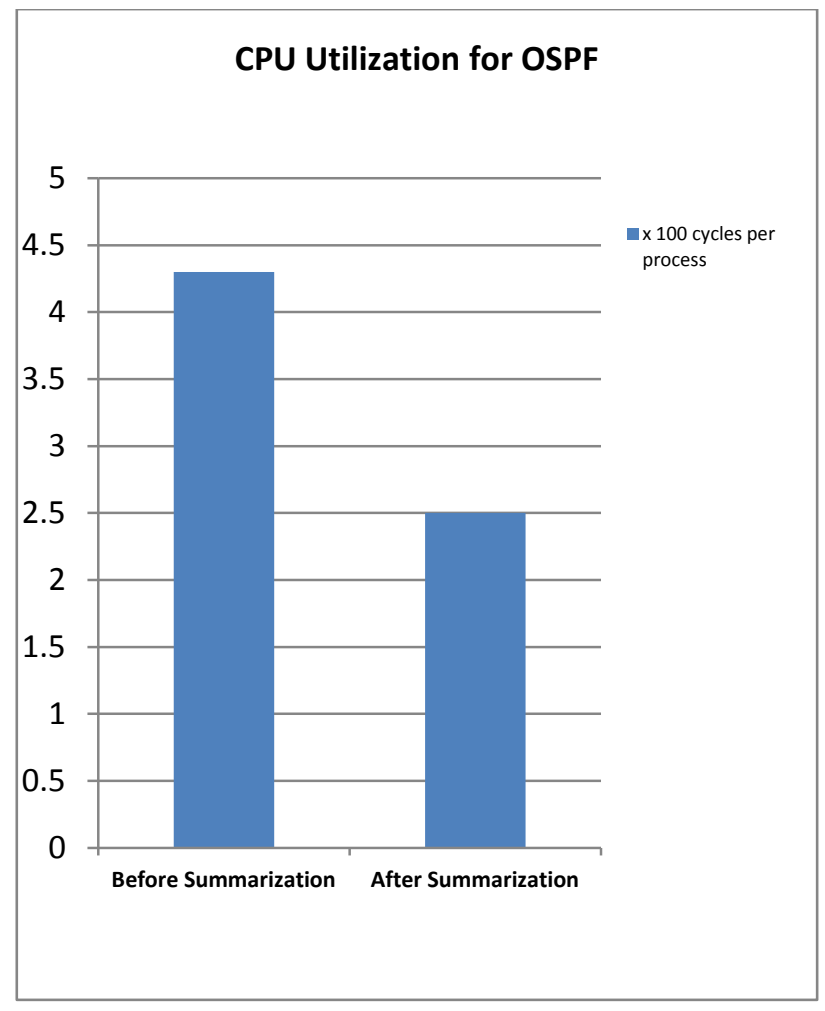

Figure 10: CPU usage in Router

The new Topology Table formed for OSPF will also has lower memory size as shown in figure 11 .

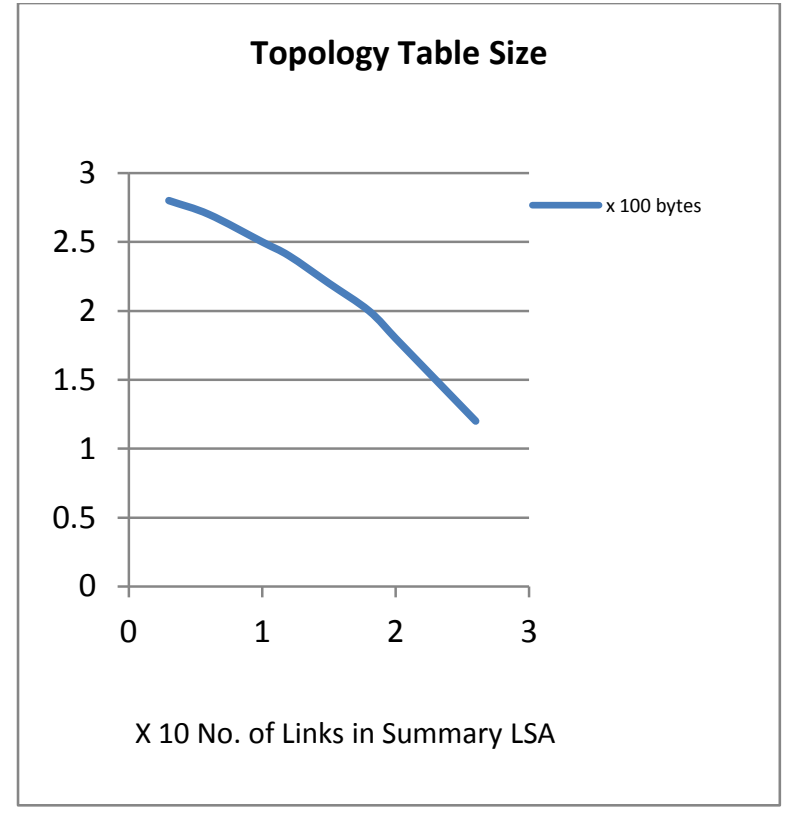

Figure 11: OSPF Database (Top. Table) size

Also the new proposed feature is easily compatible with existing OSPF working thus making it a cup cake to deal with. Thus, the result obtain after Intra-area Summarization can be listed as

1. Less CPU Utilization for OSPF process.

2. Reduced Database Size for OSPF Storage.

3. Smaller Routing Table Size for processing.

4. Better Convergence Time.

5. Less Hardware Cost requirement.

6. Easy and Flexible deployment.

\section{CONCLUSION}

As the only solution nowadays exist is to divide an OSPF autonomous system into independent routing areas, allows area topology abstraction, reducing route overhead, table size, and convergence time, while providing some isolation from bad unwanted routing data. On the contrary, areas reduce connectivity, while increasing configuration complexity, routing path length, and traffic concentration.

This paper has presented an analysis of OSPF different area configuration. The comparative analysis has been done on the same network with EIGRP. Performance has been measured on the basis of some parameters that aimed to figure out the effect of Intra-area summarization.

Thus, from the results obtained in experiments one can thoroughly conclude that our approach of Intra-area summarization not only overcome these huddles but also provides a method which is easily compatible with existing OSPF frame work. Network performance can also be enhanced by Intra-area summarization rather than increasing the number of areas.

\section{REFERENCES}

[1] Aman Shaikh, Charles Kalmanek , Dongmei Wang, Guangzhi Li and Jennifer Yates. An Efficient Algorithm for OSPF Subnet Aggregation at 11th IEEE International Conference on Network Protocols (ICNP'2013) 1092$1648 / 13$. 
[2] Aparajit Utpat, Chandan N Bhagwat and Y.Navaneeth Krishnan. Performance Analysis of OSPF Routing Protocols for Greener Internetworking at International Conference in Distributed Computing \& Internet Technology (ICDCIT-2013) Proceedings published in International Journal of Computer Applications (IJCA) $(0975-8887)$.

[3] OSPF Network Design Solutions, OSPF design covered in the Cisco Press book, (ISBN 1-57870-046-9).

[4] Mohamad A. Yehia, Mohammed S. Aziz and Hussein A. Elsayed. Analysis of IGP Routing Protocols for Real Time Applications: A Comparative Study in International Journal of Computer Applications (IJCA) (0975 - 8887) Volume 26- No.3, July 2011.

[5] Anuj Gupta and Neha Grang. Compare OSPF Routing Protocol with other Interior Gateway Routing Protocols in International Journal of Engineering, Business and Enterprise Applications (IJEBEA) IJEBEA 13-147; 2013, IJEBEA.

[6] Atul Aggarwal, Shelej Khera. Combat Resources Shortages by making Stub Areas and Route Summarization in OSPF in International Journal of Scientific and Research Publications, Volume 2, Issue 8, August 20121 ISSN 2250-3153.

[7] Albert Greenberg, Aman Shaikh, Chris Isett, Matthew Roughan and Joel Gottlieb. A Case Study of OSPF Behavior in a Large Enterprise Network.

[8] A. Shaikh , E. Baccelli, G. Choudhury, H. Hosseini, K.Trivedi , M. Goyal, and M. Soperi. Improving Convergence Speed and Scalability in OSPF at IEEE communications surveys \& tutorials. 